\title{
Calculations of the Bistatic Scattering Cross Section of a Sphere With an Impedance Boundary Condition
}

\author{
James R. Wait and Carolen M. Jackson \\ Contribution From the National Bureau of Standards, Boulder, Colo. \\ (Received September 16, 1964)
}

\begin{abstract}
Extensive numerical results for electromagnetic scattering from spheres are presented in graphical form. To simulate various types of coatings, the surface impedance of the sphere is a specified complex function. It is shown conclusively that the forward scattering lobe is almost independent of the characteristics of the coating. On the other hand, the backscattering and side scattering are profoundly modified by changes in the effective impedance of the coating.
\end{abstract}

\section{Introduction}

Scattering of electromagnetic waves from spheres is a subject which has permeated the literature for over half a century. An exhaustive survey of the subject is found in a highly recommended book by Van de Hulst [1957.] An important collection of informative papers reporting recent advances is also available [Kerker, 1964].

Despite the numerous papers treating both theoretical and experimental aspects, a number of unresolved questions remain. For example, the effect of coating conducting spheres with absorbent material is not entirely understood. Also, the related question of how an inductiveor capacitive-type surface layer modifies the scattering properties has not been answered completely. It is the purpose of this paper to present calculations for scattering from spheres under various surface impedance boundary conditions. This relatively simple model has the virtue of displaying many important features in an economical manner. This approach has been used previously by various investigators [Wait, 1956; Hiatt et al., 1960a, 1960b; Garbacz, 1964] who have treated closely related problems.

\section{Formulation}

The spherical model used as a basis for calculation is illustrated in figure 1 with reference to a Cartesian coordinate system $(x, y, z)$. A plane wave is incident from the negative $z$ direction and polarized with the electric field $E_{0}$ in the $x$ direction. With reference to a spherical coordinate system $(r, \theta, \phi)$ also centered at the sphere, the scattered field components may be written

$$
E_{\theta}^{s}=\frac{-i E_{0}}{k r} e^{-i k r}(\cos \phi) P(\theta),
$$

and

$$
E_{\phi}^{s}=\frac{i E_{0}}{k r} e^{-i k r}(\sin \phi) Q(\theta)
$$




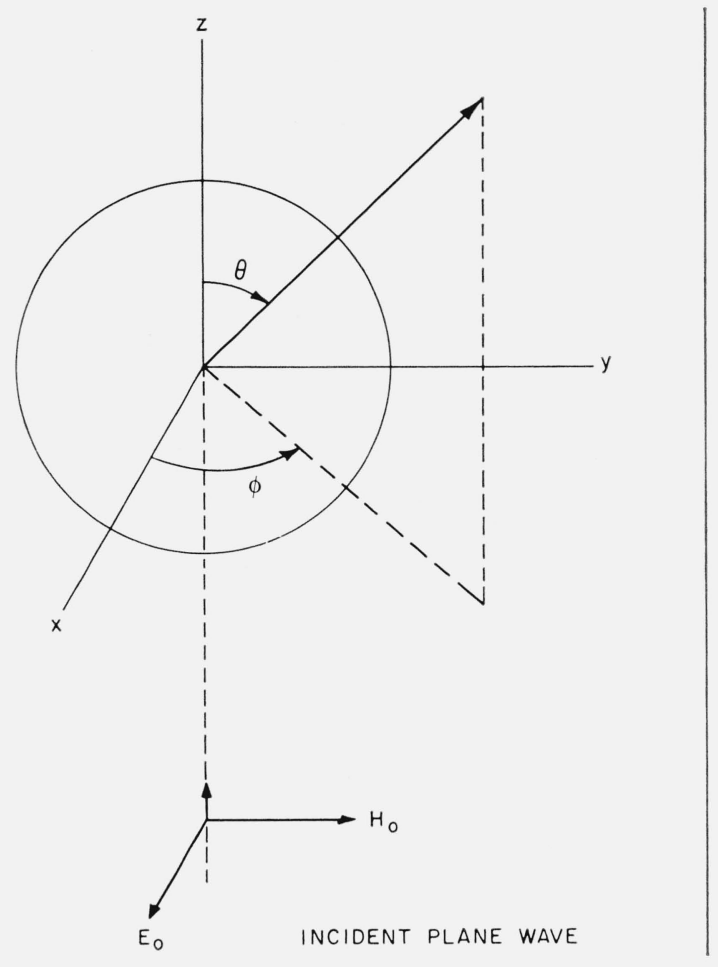

FIGURE 1. Geometry for the spherical scatterer.

where $k=2 \pi /$ wavelength and where $P$ and $Q$ are functions of $\theta$ only. The latter are defined by

$$
P(\theta)=\sum_{n=1}^{\infty} \frac{2 n+1}{n(n+1)}\left[B_{n} \frac{d}{d \theta} P_{n}^{1}(\cos \theta)+C_{n} \frac{P_{n}^{1}(\cos \theta)}{\sin \theta}\right]
$$

and

$$
Q(\theta)=\sum_{n=1}^{\infty} \frac{2 n+1}{n(n+1)}\left[B_{n} \frac{P_{n}^{1}(\cos \theta)}{\sin \theta}+C_{n} \frac{d}{d \theta} P_{n}^{1}(\cos \theta)\right]
$$

where the coefficients $B_{n}$ and $C_{n}$ are a function of $k a$, the circumference of the sphere in wavelengths, and the surface impedance $Z$ of the sphere. The explicit definition of these coefficients $B_{n}$ and $C_{n}$ is given in the appendix where the derivation is outlined. The function $P_{n}^{1}(\cos \theta)$ is the associated Legendre function of order $n$ and index 1.

As indicated above, the solution for the present problem is expressed in terms of the surface impedance $Z$ which requires that the total tangential fields at $r=a$ are related as follows:

$$
E_{\theta}=-Z H_{\phi} \text { and } E_{\phi}=Z H_{\theta}
$$

These are often termed the Leontovich boundary conditions when applied to the interface between free space and a highly conducting medium [e.g., ch. 2 in Wait, 1962]. Actually, they are a valid representation for other type surfaces such as a dielectric coated conductor or a finely corrugated surface, in which cases $Z$ is highly inductive. In the general case, such as for a radially inhomogeneous sphere, it is necessary to recognize that surface impedances are dependent on mode number.

For the present discussion, it is assumed that the boundary may be characterized by a constant surface impedance $Z$. This is conveniently normalized by writing

$$
Z / \eta_{0}=i G
$$


where $\eta_{0}=120 \pi$ is the intrinsic impedance of free space and $G$ is a dimensionless factor. For a purely inductive surface, $G$ is positive real, while for a capacitive surface, $G$ is negative real. If the sphere is composed of a homogeneous, highly conducting material,

$$
G \cong \frac{1-i}{\sqrt{2}}|G|
$$

where $|G|^{2}=\epsilon_{0} \omega / \sigma_{g}$ in terms of the conductivity $\sigma_{g}$ of the sphere, the dielectric constant of free space $\epsilon_{0}$, and the angular frequency $\omega$. The restrictions inherent in the use of the surface impedance require that $|G|^{2}<<1$ [Wait, 1962] in (7).

In the general case of a surface impedance boundary, it is desirable to write

$$
Z=|Z| e^{i \Phi}
$$

or

$$
G=-i|G| e^{i \Phi}
$$

where $\Phi$ is a phase angle. For the inductive boundary $\Phi=90^{\circ}$, for the capacitive boundary $\Phi=-90^{\circ}$, whereas, for the homogeneous conductor $\Phi=45^{\circ}$. It might be mentioned that with various forms of concentrically stratified conducting spheres $\Phi$ may be anywhere in the range 0 to $90^{\circ}$. Furthermore, certain types of corrugated surfaces loaded with lossy material could give rise to effective surface impedances which have phase angles also in this range [Wait, 1962].

To present numerical results in an economical manner, two bistatic radar cross sections $\sigma_{\epsilon}(\theta)$ and $\sigma_{h}(\theta)$ are introduced which are for $E$-plane and $H$-plane scattering. Explicitly, these are defined by:

$$
\left.\sigma_{e}(\theta)=\frac{4 \pi r^{2}\left|E_{\theta}\right|^{2}}{E_{0}^{2}}\right]_{\phi=0}
$$

and

$$
\left.\sigma_{h}(\theta)=\frac{4 \pi r^{2}\left|E_{\phi}\right|^{2}}{E_{0}^{2}}\right]_{\phi=90^{\circ}}
$$

Using (1) and (2), and dividing by the geometrical cross section, it is seen that (10) and (11) may be written

$$
\frac{\sigma_{e}(\theta)}{\pi a^{2}}=\frac{4}{(k a)^{2}}|P(\theta)|^{2}
$$

and

$$
\frac{\sigma_{h}(\theta)}{\pi a^{2}}=\frac{4}{(k a)^{2}}|Q(\theta)|^{2}
$$

A computer program was devised for the functions $P(\theta)$ and $Q(\theta)$. The spherical Bessel functions which are involved in the coefficients $B_{n}$ and $C_{n}$ were computed by a method due essentially to Miller [1950]. To secure convergence of the series expansions for $P(\theta)$ and $Q(\theta)$, at least $2 \mathrm{ka}$ terms were used in the summation. For the graphical accuracy shown in the figures, this was more stringent than necessary.

\section{Description of Results}

The numerical results for the two bistatic cross sections are plotted as a function of $\theta$ for a wide variety of situations. The basic parameters are the circumference, $k a$, of the sphere in wavelengths and the complex value of $G$. 


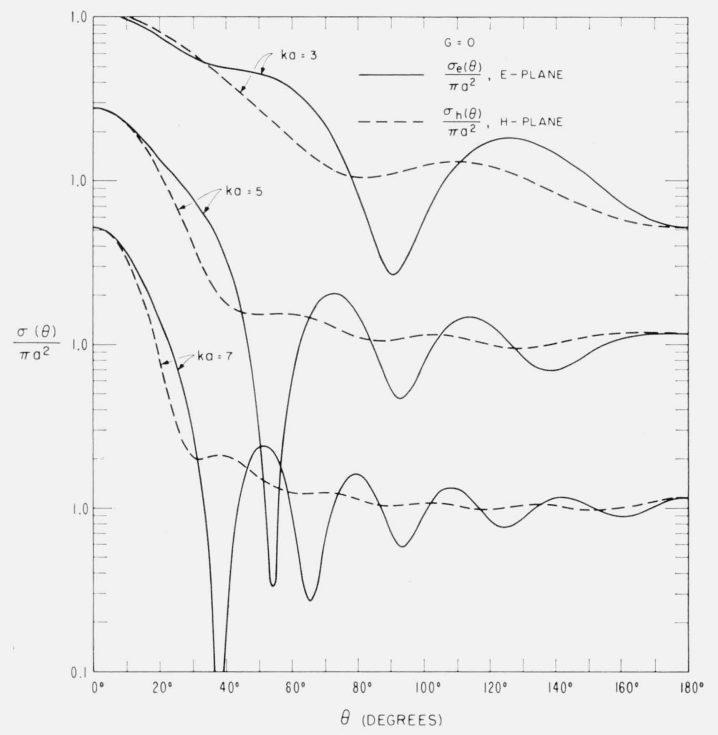

Figure 2a.

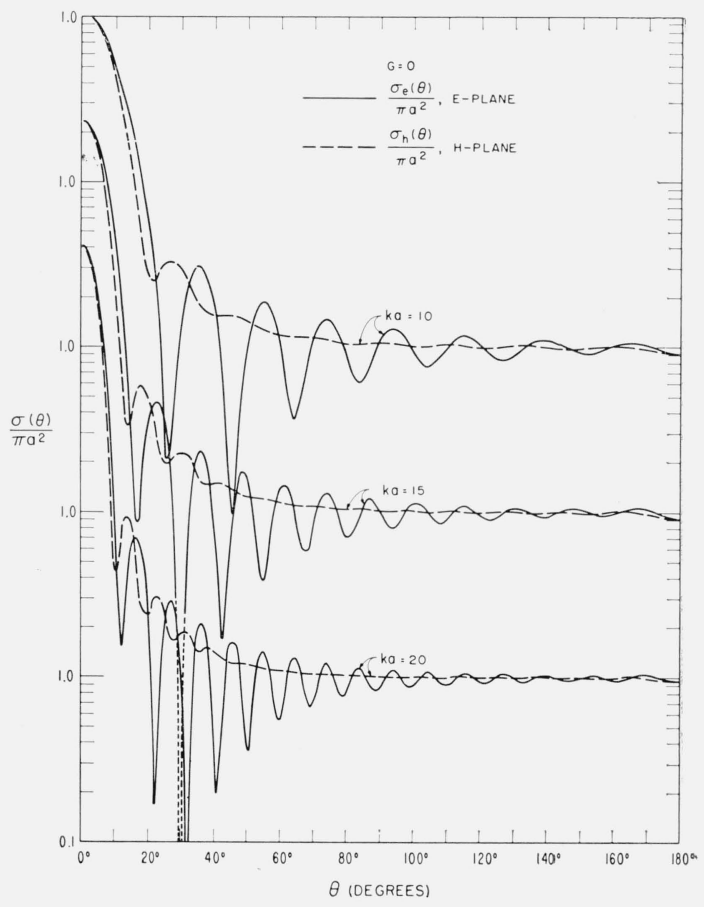

Figure 2b.

In figures $2 \mathrm{a}$ and $2 \mathrm{~b}$, the cross sections are plotted for $k a$ values ranging from 3 to 20 under the condition $G=0$ (i.e., perfectly conducting sphere). These particular curves may be compared directly with similar plots prepared by Nelson Logan ${ }^{1}$ and colleagues. Where the $k a$. values are common the data are identical within the graphical accuracy. This would appear to be a good check on the computer program for this special case.

It is evident from the curves in figures $2 \mathrm{a}$ and $2 \mathrm{~b}$ that the forward scattering (i.e., corresponding to $\theta \cong 0$ ) is greatly enhanced over the backscattering. In fact, the (normalized) forward scattering cross sections both become approximately equal to $(k a)^{2}$ while the (normalized) backscatter is approximately unit. Thus, in the forward direction, the sphere is behaving as a "black disk," particularly for the larger $k a$ values. On the basis of Huygen's principle, it follows from Van de Hulst [1957] that for small values of $\theta$,

$$
\sigma_{e}(\theta) \cong \sigma_{h}(\theta) \cong(k a)^{2} \iint e^{-i k(x \cos \phi+y \sin \phi) \sin \theta} d x d y,
$$

where the integration is over the area of the "black disk." In the case of the sphere or its equivalent circular black disk, the integrations are readily carried out to give

$$
\frac{\sigma_{e}(\theta)}{\pi a^{2}} \cong \frac{\sigma_{h}(\theta)}{\pi a^{2}} \cong(k a)^{2}\left[\frac{2 J_{1}(k a \sin \theta)}{(k a \sin \theta)}\right]^{2}
$$

For pure forward scattering (i.e., $\theta \rightarrow 0$ ), the square bracketed term involving the $J_{1}$ Bessel function approaches unity which is a broad maximum. It decreases smoothly to zero at $\theta=\theta_{0}$ when $J_{1}\left(k a \sin \theta_{0}\right)=0$ or when $k a \sin \theta_{0}=3.832$. For $k a=10,15$, and 20 , the corresponding. values of $\theta_{0}$ are $22.5^{\circ}, 14.8^{\circ}$, and $11.0^{\circ}$. These correspond quite well to the first minima in the calculated curves (fig. 2b) for $\sigma_{e}(\theta)$ and $\sigma_{h}(\theta)$ in the forward direction. The agreement is better for the larger $k a$ values, as expected. 
Because of the crude physical assumptions underlying the application of Huygen's principle, it is not expected that (15) should be used for angles except near forward scattering. Furthermore, being a scalar theory, it does not distinguish between the two wave polarizations. However, this simple "black disk" model is particularly appropriate for describing the shape and width of the forward main lobe in the scattering pattern. A further demonstration is to employ (15) to calculate the values of $\theta=\theta_{1}$ where $\sigma_{e}(\theta)$ and $\sigma_{h}(\theta)$ drop to $1 / 2$ of the central maximum at $\theta=0$. This condition is $k a \sin \theta_{1}=1.61$. For $k a=10,15$, and 20 , the corresponding values of $\theta_{1}$ are found to be $9.3^{\circ}, 6.1^{\circ}$, and $4.6^{\circ}$ which, within two significant figures are identical to the curves in figure $2 \mathrm{~b}$ computed from the exact series solutions.

It is evident that the curves in both figures $2 \mathrm{a}$ and $2 \mathrm{~b}$ are highly oscillatory. This results from the well-known effect of the "creeping waves." In other words, the two waves diffracted around the sphere interact with one another to form an interference pattern. This effect has been discussed for perfectly conducting spheres by Van de Hulst [1957], Belkina and Weinstein [1957], Logan and Yee [1961], and many others. It is further remarked here that the interference pattern is much more pronounced for $E$-plane scattering than it is for $H$-plane scattering. For perfectly conducting spheres, this is also a very well-known effect and is primarily a consequence of relatively low attenuation of the creeping wave in the E-plane case.

For perfectly conducting spheres, it is finally remarked that backscattering cross sections, for the larger $k a$ values, become very close to the geometrical cross section $\pi a^{2}$. This is particularly evident for the curves in figure $2 \mathrm{~b}$, as remarked above.

The effect of choosing a succession of real positive $G$ values is illustrated in figures 3a to $5 \mathrm{~b}$ for the same range of $k a$ values used for the perfectly conducting spheres (i.e., $G=0$ ). As mentioned before, a purely inductive boundary is described by real positive $G$. It is very evident from these curves that, even for the largest $G$ value chosen, the magnitude and shape of the forward-scatter lobe are not changed markedly. Furthermore, the magnitude of the backscattering cross section is only slightly modified from the corresponding curves for $G=0$. The only significant changes in the curves in figures $3 \mathrm{a}$ to $5 \mathrm{~b}$ from those in figures $2 \mathrm{a}$ and $2 \mathrm{~b}$ are the enhancement of the ripples in the $E$-plane scattering pattern. This is consistent with the expected reduction of the attenuation of the creeping wave which results from an inductive-type coating [Wait, 1964].

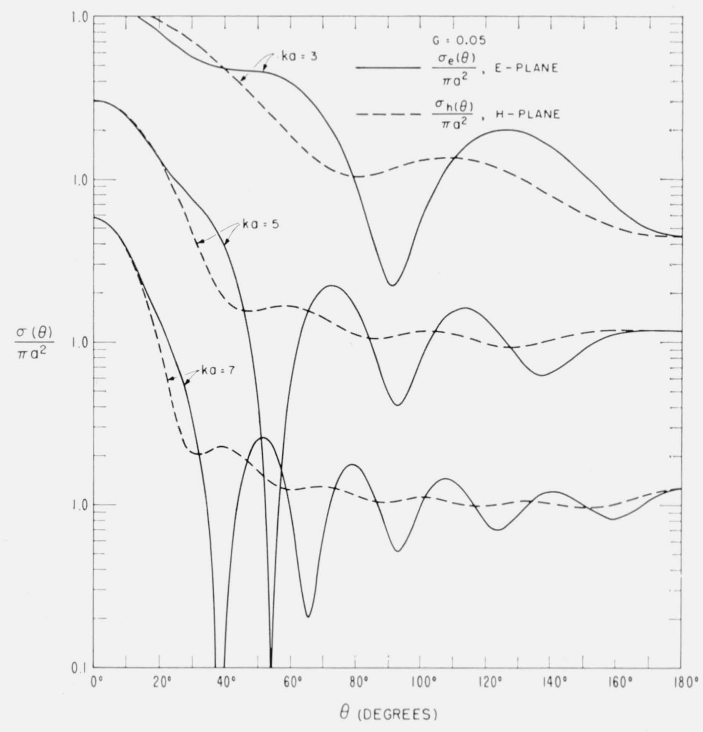

Figure 3a.

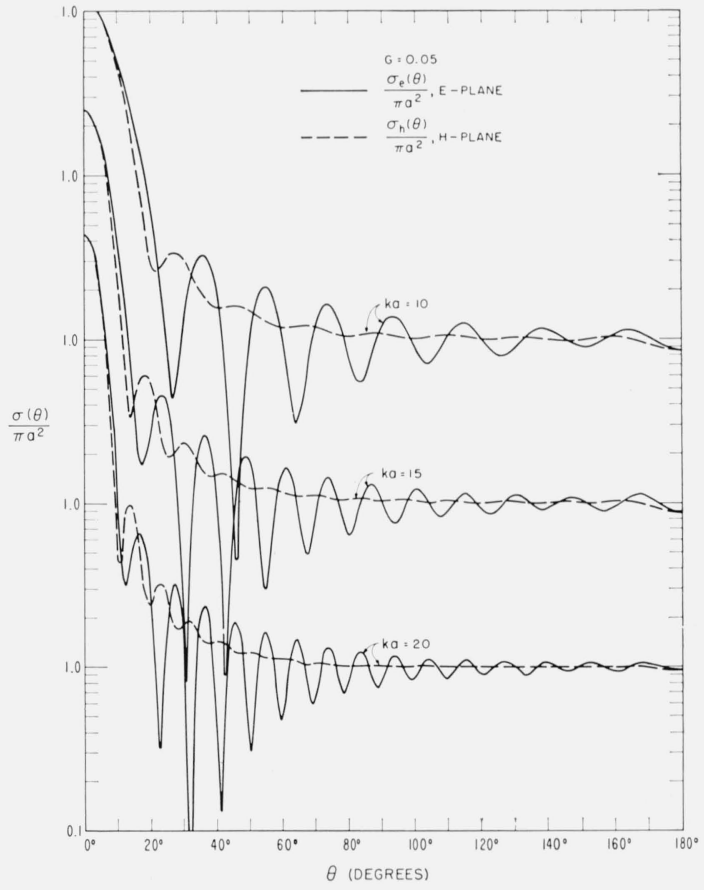

Figure $3 b$. 


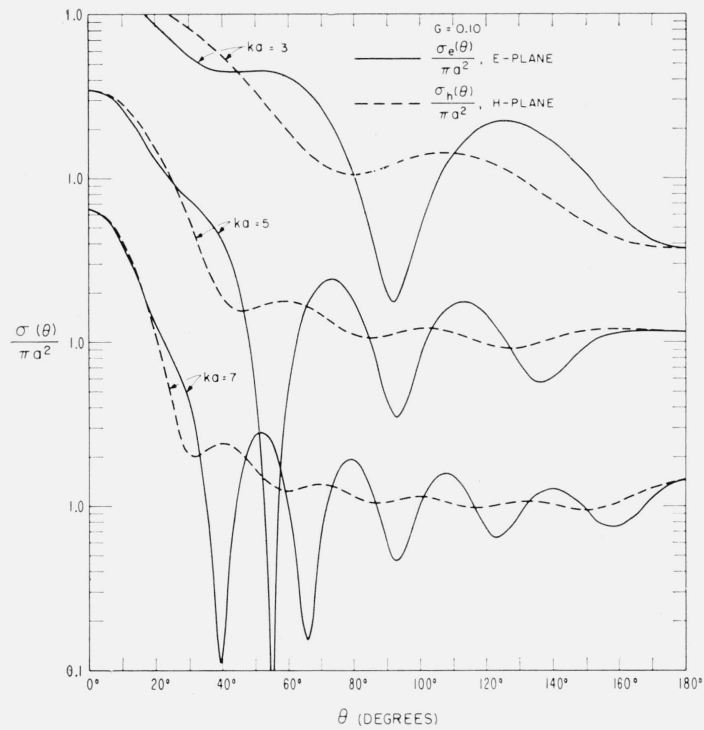

Figure 4 a.

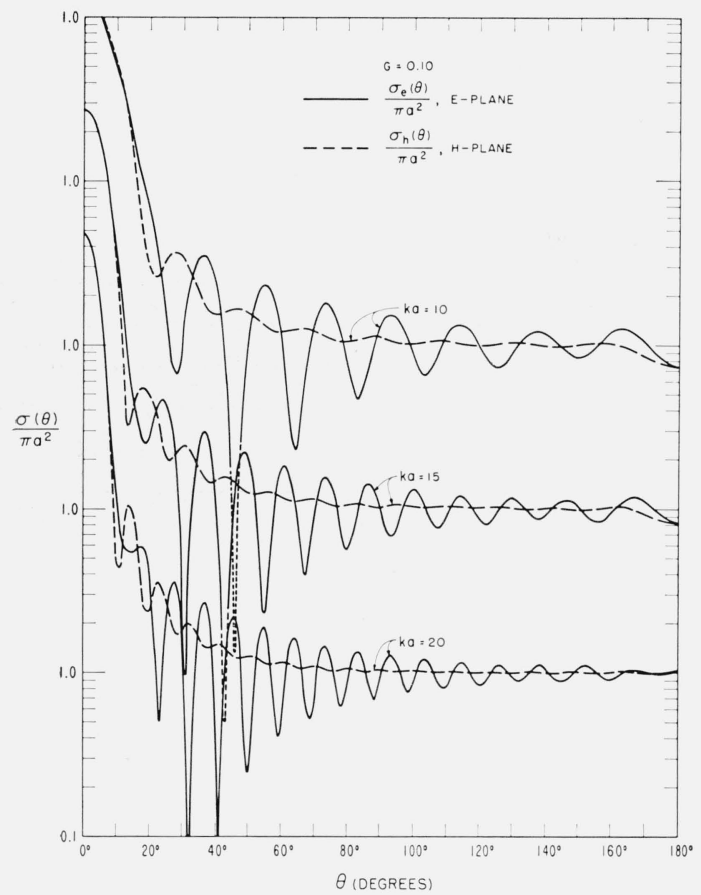

Figure $4 \mathrm{~b}$.

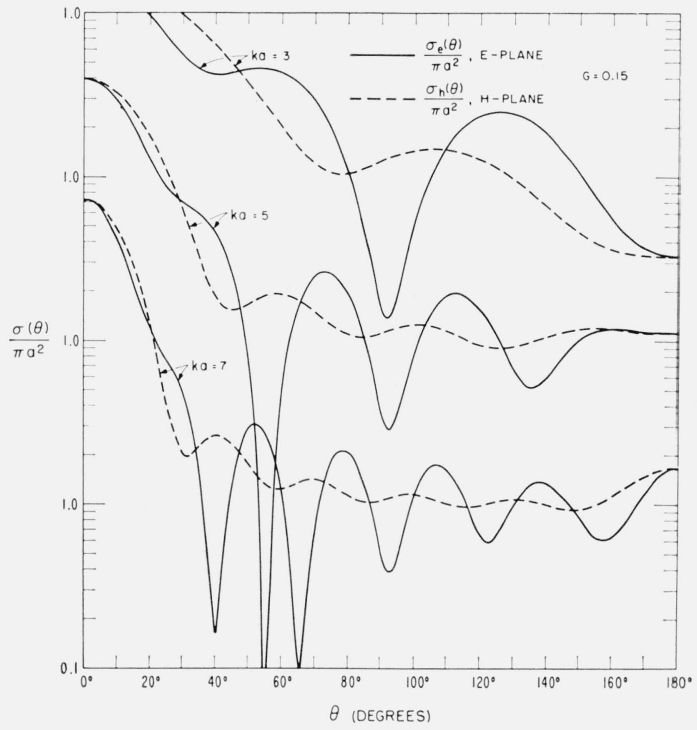

Figure 5a.

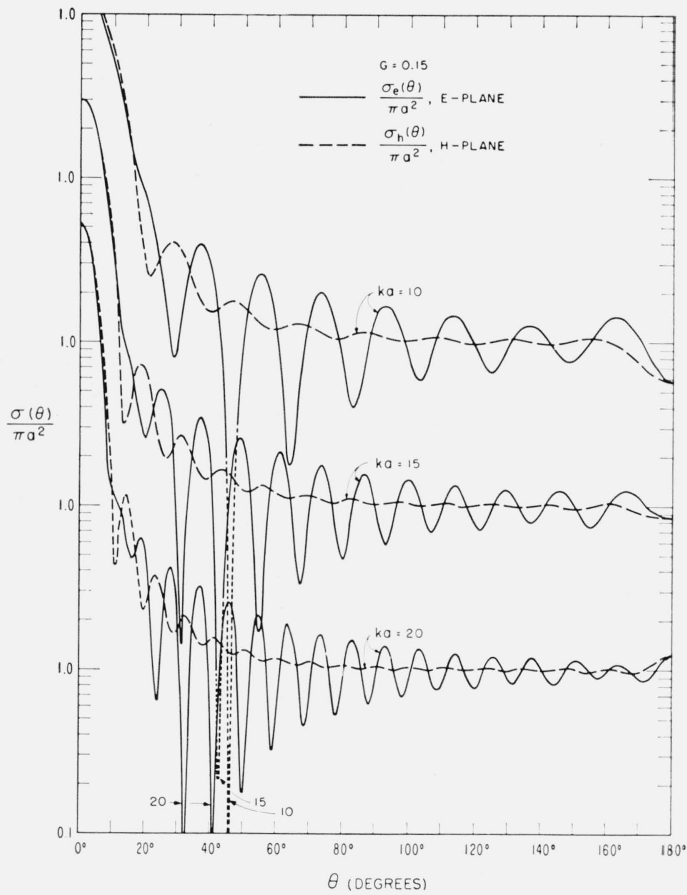

FiguRe 5b.

\section{Digression on Fresnel Reflection Coefficients}

In discussing the numerical results for backscattering from spheres with impedance boundary layers, it is useful to consider first what one may expect on the basis of geometrical optics. The concept is extremely simple; it is assumed that the cross sections $\sigma_{e}$ and $\sigma_{h}$ are individually proportional to $\left|R_{e}\right|^{2}$ and $\left|R_{h}\right|^{2}$ where the latter are the square of the Fresnel reflection coefficients for $E$-plane and $H$-plane scattering, respectively. Thus, within this geometrical-optical approximation,

$$
\frac{\sigma_{e}}{\pi a^{2}} \cong\left|R_{e}\right|^{2} \text { and } \frac{\sigma_{h}}{\pi a^{2}} \cong\left|R_{h}\right|^{2} .
$$


The reflection coefficients are defined by [Wait, 1962],

$$
R_{e}=\frac{C-\Delta}{C+\Delta} \text { and } R_{h}=\frac{C \Delta-1}{C \Delta+1}
$$

where $\Delta=Z / \eta_{0}=i G$ is the normalized surface impedance and $C=\cos \beta=\sin (\theta / 2)$ where $\beta$ is the local angle of incidence for the specular ray.

In terms of the magnitude $|G|$ and the phase angle $\Phi$, it is seen that, according to geometrical optics,

$$
\frac{\sigma_{e}}{\pi a^{2}} \sim \frac{[\sin (\theta / 2)-|G| \cos \Phi]^{2}+[|G| \sin \Phi]^{2}}{[\sin (\theta / 2)+|G| \cos \Phi]^{2}+[|G| \sin \Phi]^{2}}
$$

and

$$
\frac{\sigma_{h}}{\pi a^{2}} \sim \frac{[|G| \cos \Phi \sin (\theta / 2)-1]^{2}+[|G| \sin \Phi \sin (\theta / 2)]^{2}}{[|G| \cos \Phi \sin (\theta / 2)+1]^{2}+[|G| \sin \Phi \sin (\theta / 2)]^{2}} .
$$

It is rather interesting to note that when $G$ is real (i.e., $\Phi=90^{\circ}$ ), the right-hand sides of the preceding two equations are unity. This is not really surprising as reflection takes place without loss from a purely reactive surface. This is also true, of course, when $|G|=0$, which corresponds to the perfectly conducting surface.

\section{Further Description of Results and Comparisons With Geometric Optics}

It is now worth while to return and examine the various curves in figures $2 \mathrm{a}$ to $5 \mathrm{~b}$. It is evident that for the larger $k a$ values (i.e., 10, 15, and 20) and for $\theta$ in the range $90^{\circ}$ to $180^{\circ}$, $\sigma_{e} / \pi a^{2}$ and $\sigma_{h} / \pi a^{2}$ are within the range 0.6 to 1.5. As expected, of course, geometric optics ignores the diffracted or creeping waves.

To illustrate a much more favorable comparison between the exact theory and geometrical optics, we choose a surface impedance which is purely real. Thus, $Z=|Z|$ and $G=-i|G|$ so that the phase angle $\Phi=0$. For this situation, the scattering patterns are shown in figures $6 \mathrm{a}$ and $6 \mathrm{~b}$ for the $E$-plane and $H$-plane, respectively. The values of $G$ are indicated on the curves. The close agreement between the geometrical-optical calculations and the exact theory is clearly evident. It is particularly interesting to note that when $|G|=1.00$, the backscattering according to geometric optics, is identically zero. In other words, the surface impedance matches the intrinsic impedance of free space. The exact theory for the sphere (with $k a=15$ ) shows that the backscattering cross section is less than $10^{-5}$. It may also be observed in figures $6 \mathrm{a}$ and $6 \mathrm{~b}$ that deep nulls occur whenever $|G|=\sin \theta / 2$ in the case of $E$-plane scatter and whenever $1 /|G|=\sin \theta / 2$ in the case of $H$-plane scatter. Under these conditions, the Fresnel reflections have a zero and the corresponding angle $(\theta / 2)$ is the conventional Brewster angle.

The curves in figures 7 a to $9 \mathrm{~b}$ illustrate the behavior of the cross sections for values of $G$ which are near $-i$. It is seen in figures $7 \mathrm{a}$ and $7 \mathrm{~b}$ that even for a $k a$ value as small as 3 , almost perfect absorption takes place in the backscatter direction if $G=-i$. However, very small changes in the magnitude of $G$ result in an appreciable backscatter signal with a subsequent shift of a deep null to another angle. Similar effects are seen in figures $8 \mathrm{a}, 8 \mathrm{~b}, 9 \mathrm{a}$, and $9 \mathrm{~b}$ for larger $k a$ values. In all cases, the forward scattering lobe is virtually unchanged even when the sphere has no backscatter. This is in agreement with the results of Garbacz [1964] for a similar situation.

In figures 10a to $10 \mathrm{e}$, cross-section curves are shown to illustrate the effect of varying the magnitude of $G$ over fairly wide limits. Both $E$ - and $H$-plane cases are shown for $k a$ values of five and fifteen. In figure $10 \mathrm{a}$, the value of $G$ is +0.50 , which corresponds to a highly inductive surface. Here, there is evidence of strong standing waves for the E-plane pattern for the whole range of $\theta$. As explained before, this results from the low attenuation of the creeping waves for the TM (transverse magnetic) waves. On the other hand, the standing waves for the $H$-plane pattern are only slightly enhanced by the inductive coating except for 


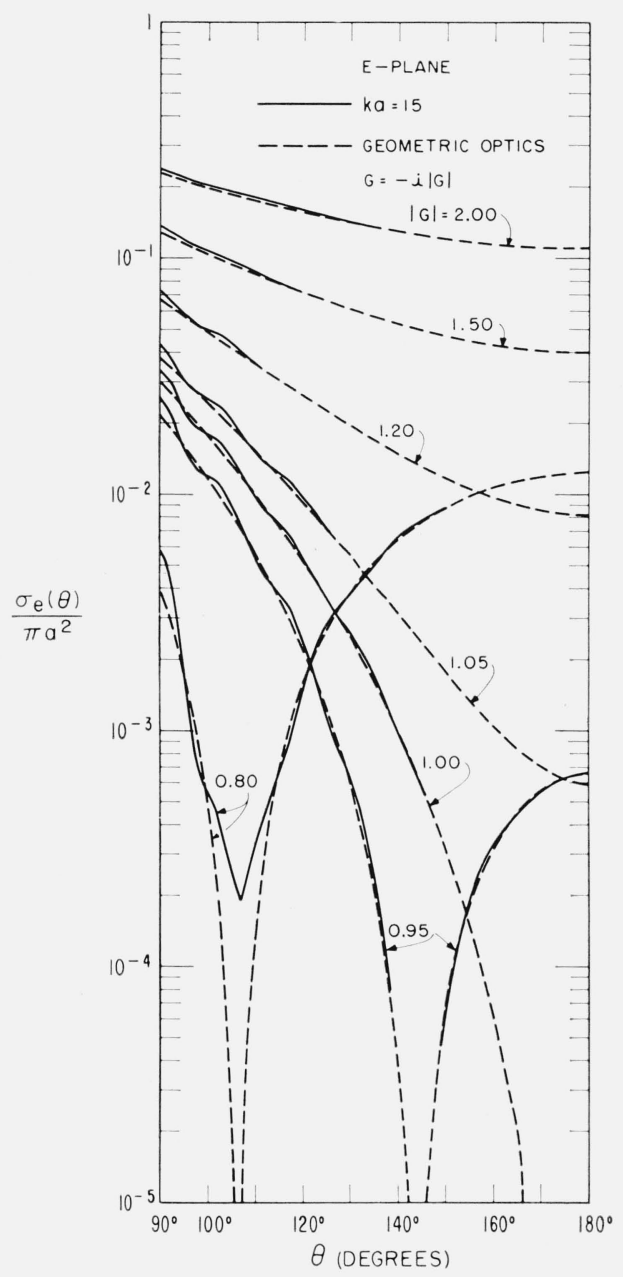

Figure 6a.

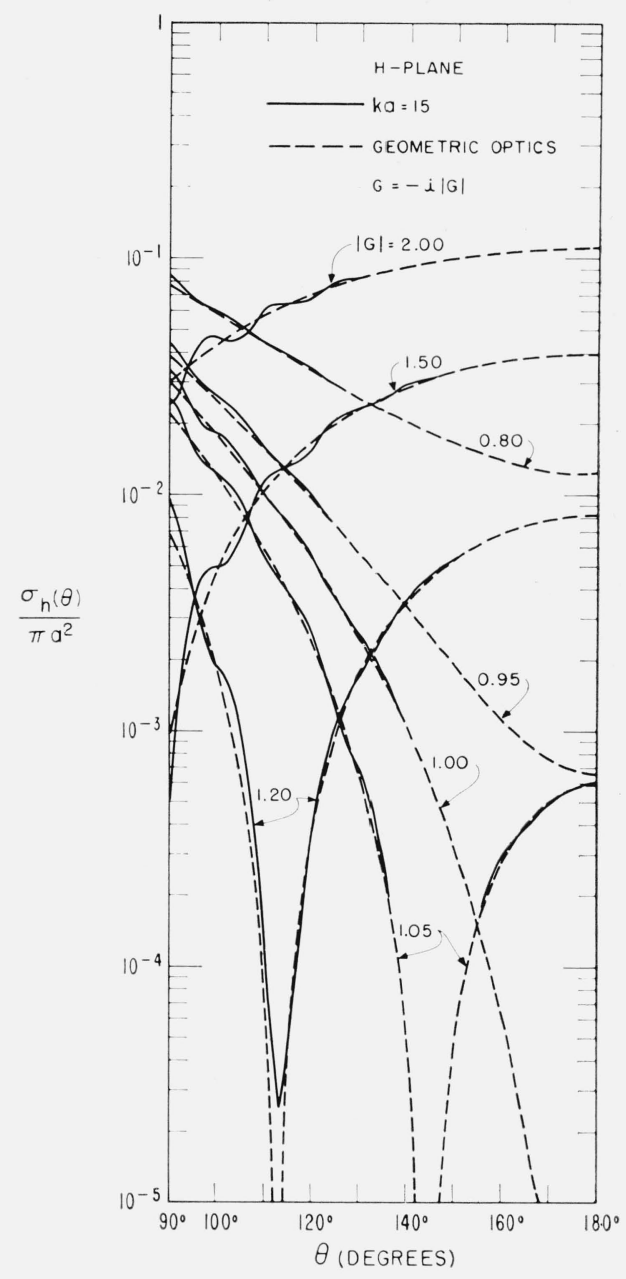

FiguRE 6 b.

directions near backscatter. As seen from figure 10b, the effects of inductive coating diminish as $G$ is reduced from 0.5 to 0.3 . Then, of course, as $G$ is reduced to zero, the corresponding curves would be identical to those shown in figures $2 \mathrm{a}$ and $2 \mathrm{~b}$.

A capacitive-type coating corresponds to taking negative real values of $G$. Cross-section patterns are shown in figures $10 \mathrm{c}, 10 \mathrm{~d}$, and $10 \mathrm{e}$ for $G=-0.1,-0.3$, and -0.5 , respectively. Here, it is interesting to note that the ripples are very small for both $E$ - and $H$-plane patterns. At the same time, the forward scattering lobe is virtually unaffected by changes of $G$ right through the range from +0.5 to -0.5 .

In figures 11a to 11c, cross-section curves are shown to illustrate the effect of a conductivetype coating material such that $Z=|Z| \exp (i \pi / 4)$ or $G=(1-i)|G| / \sqrt{2}$ or $\Phi=45^{\circ}$ (which are all equivalent statements). It is immediately evident that such a coating has the important effect of reducing the scattering cross sections for all directions except in the forward direction. Furthermore, the standing wave patterns are generally decreased by the presence of the coating. Comparisons between geometric optics and the exact theory for conductive-type coatings are shown in figures $12 \mathrm{a}$ and $12 \mathrm{~b}$ for $\theta$ in the range from $90^{\circ}$ to $180^{\circ}$. The agreement is reasonably good. 


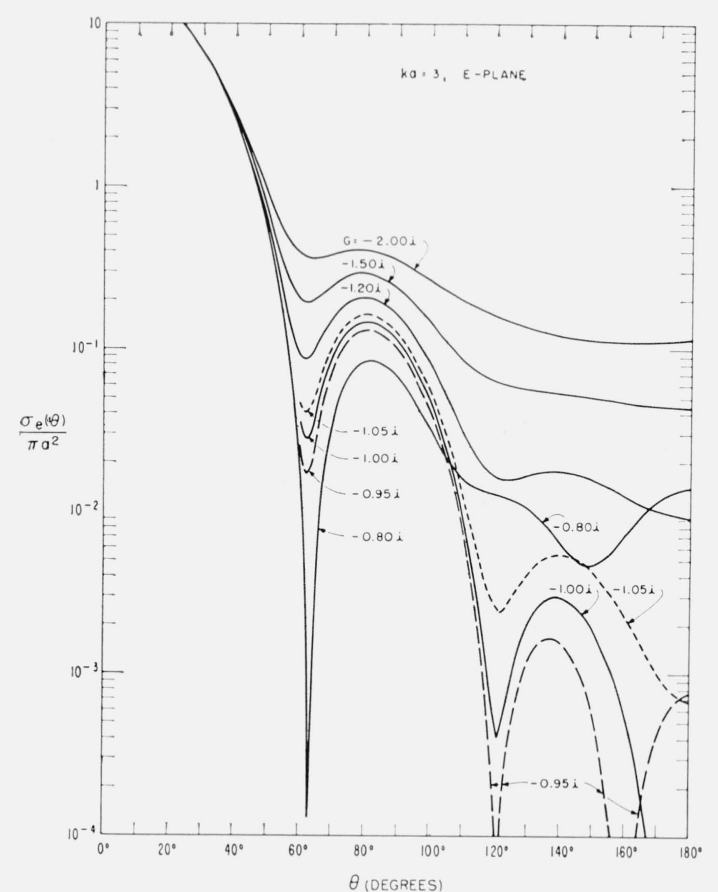

Figure 7a.

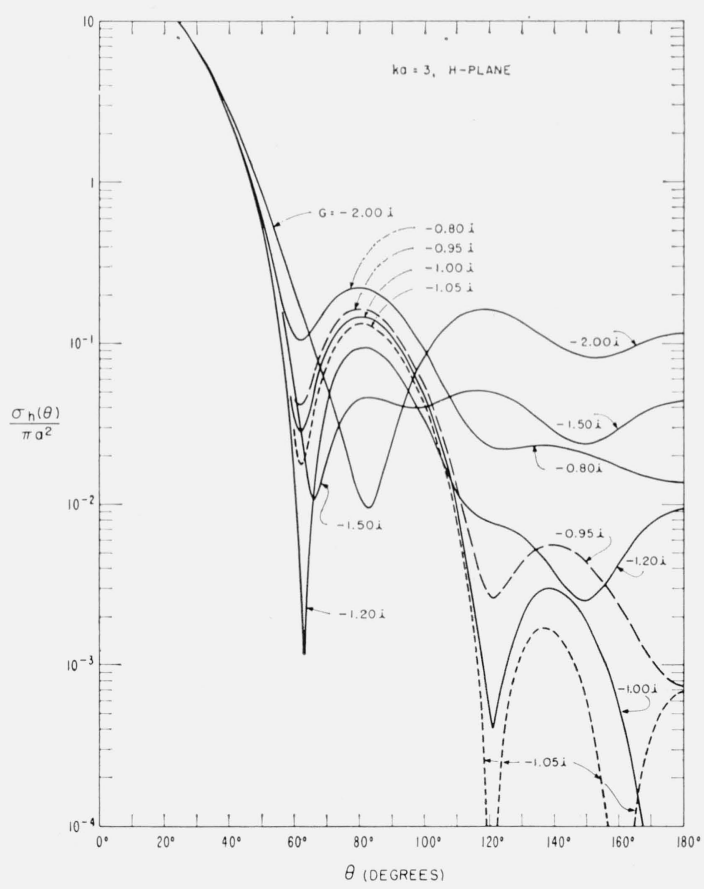

Figure $7 \mathrm{~b}$.

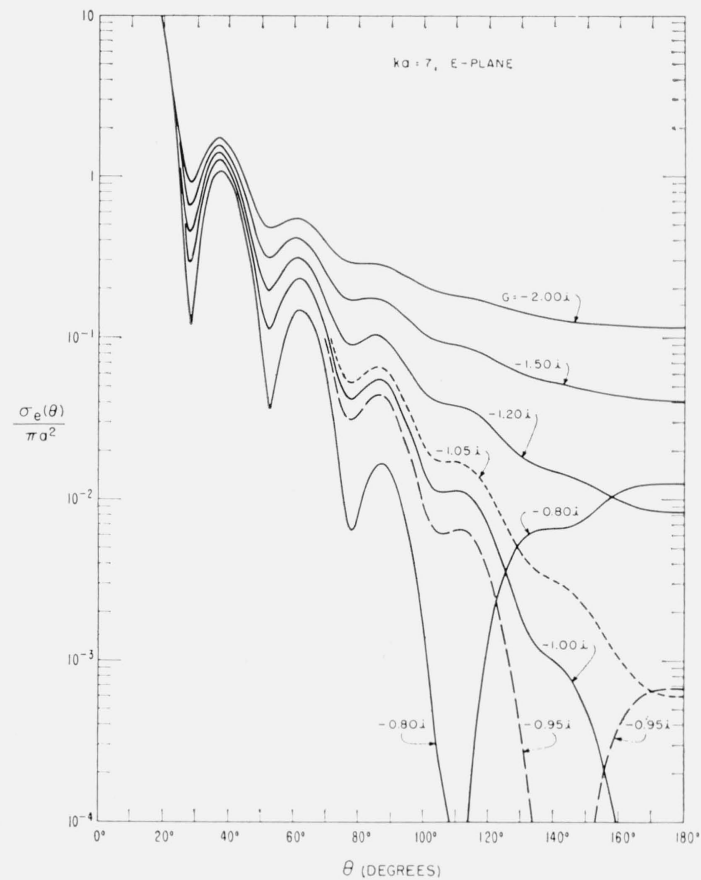

Figure $8 a$.

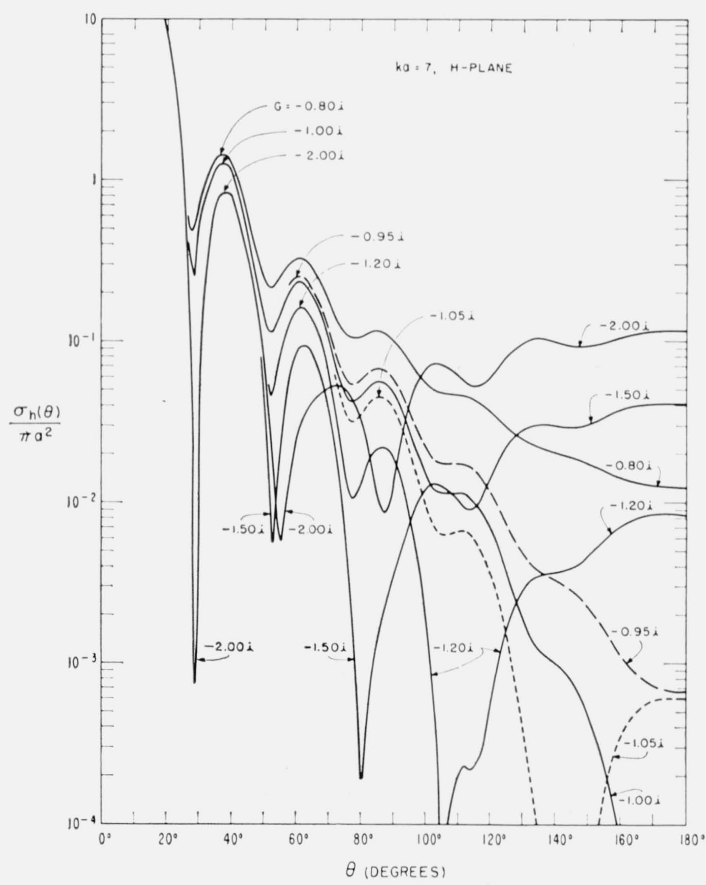

Figure $8 \mathrm{~b}$.

The effect of varying the phase angle $\Phi$ is illustrated for $k a=5$ and $|G|=0.10$ in figures $13 \mathrm{a}$ and $13 \mathrm{~b}$ for $E$-plane scatter and in figures $14 \mathrm{a}$ and $14 \mathrm{~b}$ for $H$-plane scatter. Those curves show quite clearly that the greatest reduction in scattering takes place when the surface impedance is purely real (i.e., $\Phi=0^{\circ}$ ), although marked reductions also occur when the coating is of a conductive type (i.e., $\Phi=45^{\circ}$ ). Similar-type curves are shown in figures $15 \mathrm{a}$ to $16 \mathrm{~b}$ where $k a=15$, but otherwise the conditions are the same. 


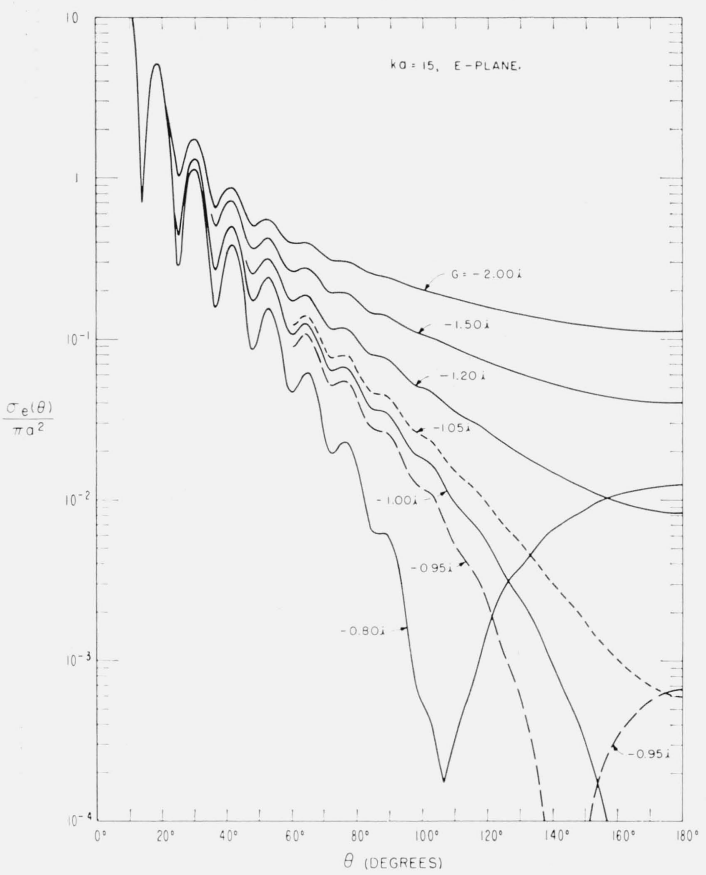

Figure 9a.

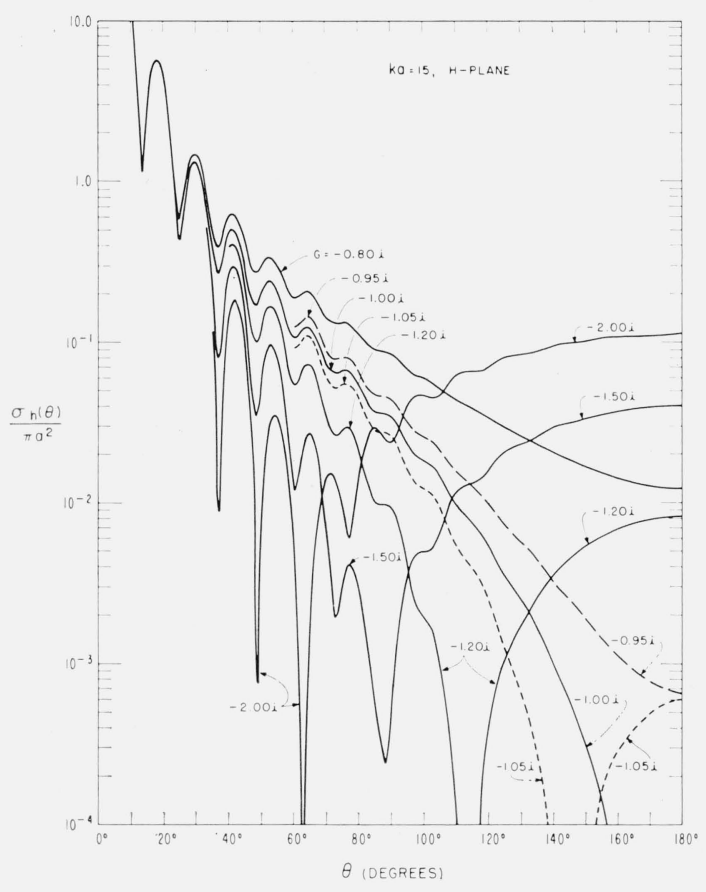

Figure $9 \mathrm{~b}$.

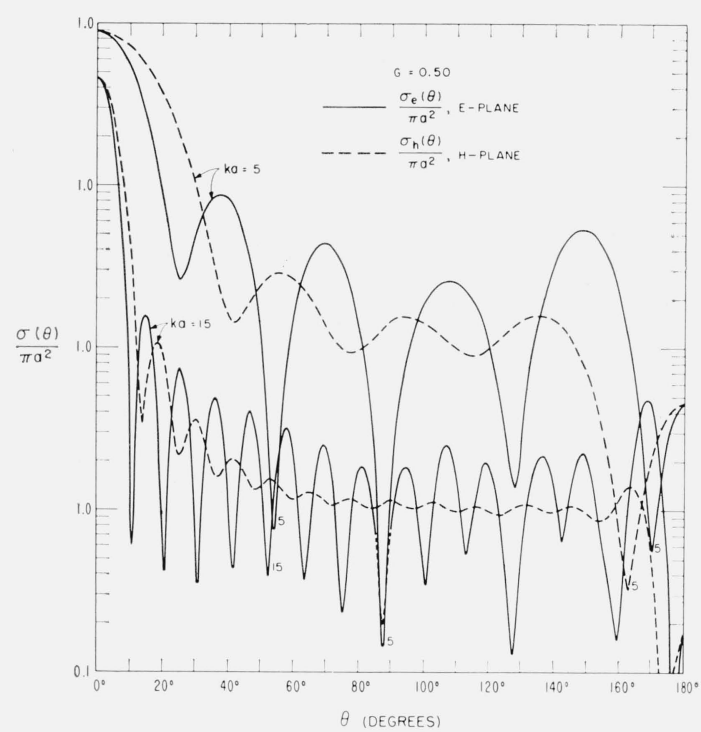

Figure 10a.

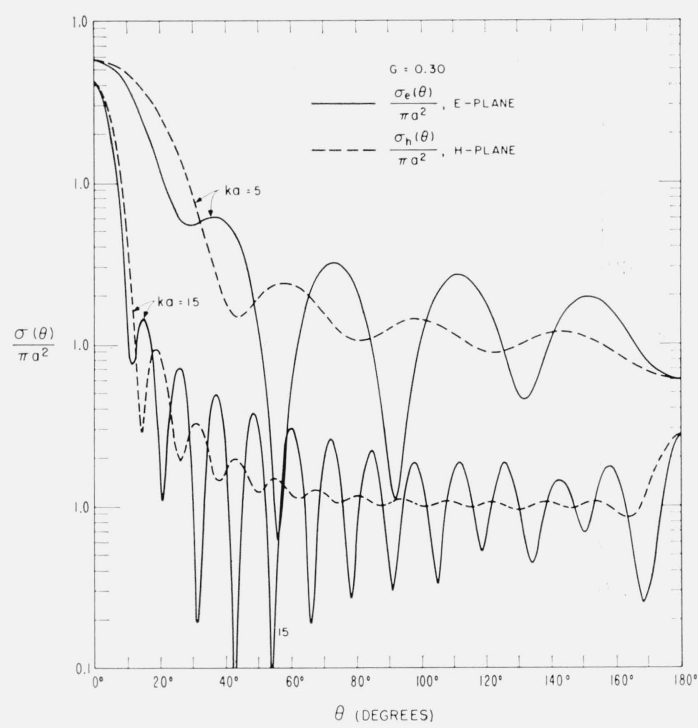

Figure 10 b.

\section{Conclusions}

The numerical results presented graphically in this paper should provide some insight into the nature of scattering from coated spheres. Of principal importance is the fact that forward scattering is hardly influenced at all by the presence of reactive or lossy-type coatings. On the other hand, the magnitude of the backscattering cross sections is significantly "modified by the magnitude and phase of the effective surface impedance of the coating. 


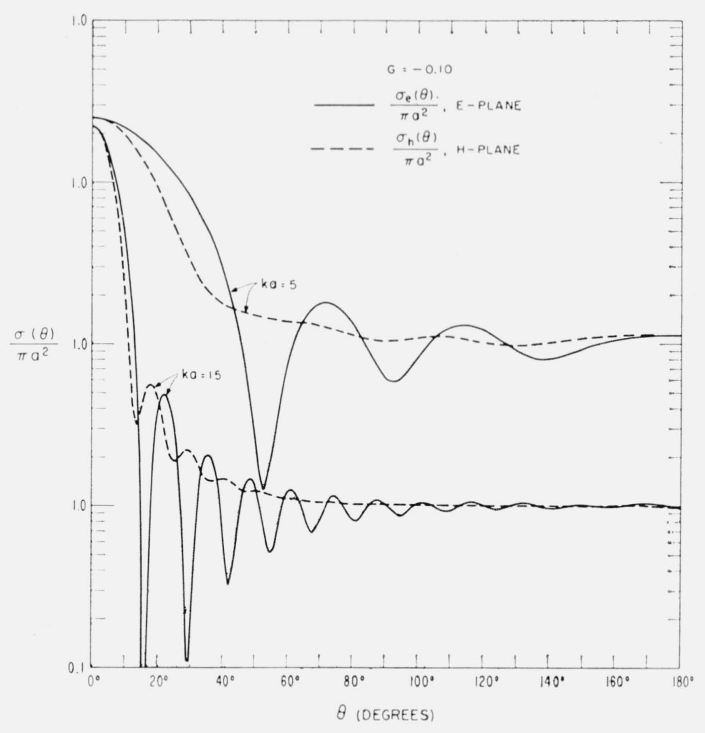

Figure 10c.

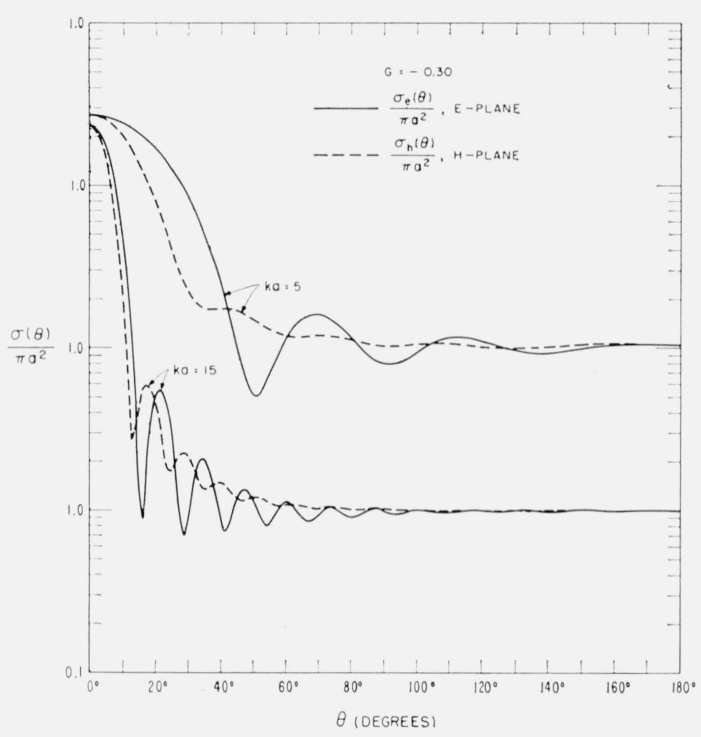

Figure 10d.

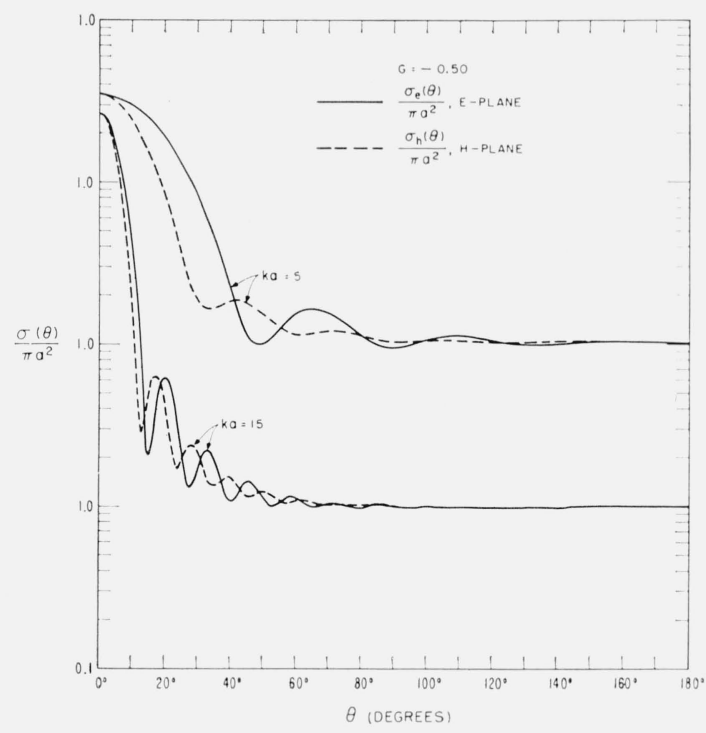

Figure 10e.

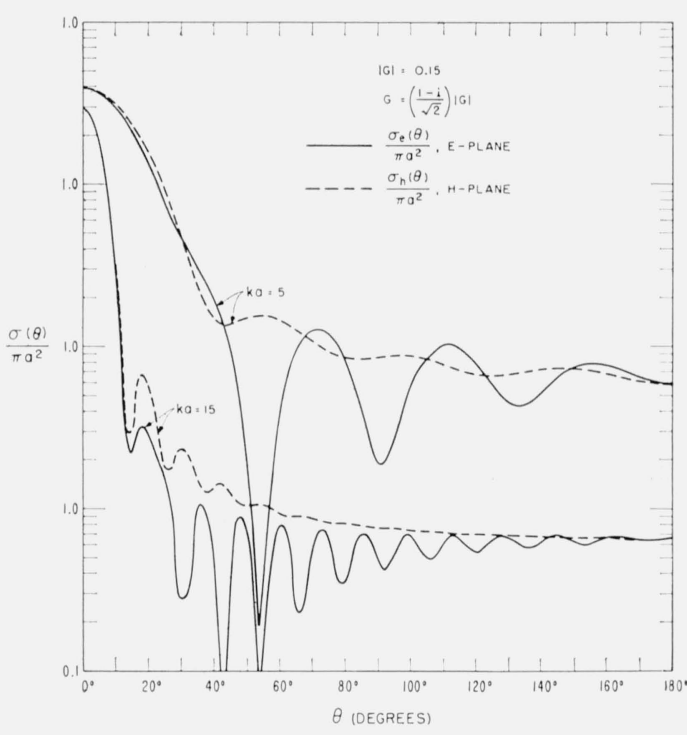

Figure 11a.

\section{Appendix. Outline" of [Solution for Scattering of a Plane Wave From a Sphere With"Impedance Boundary Conditions}

The geometry of the problem is defined in terms of spherical coordinates $(r, \theta, \phi)$ and common Cartesian coordinates $(x, y, z)$ with common origin and common polar axis as indicated in figure 1. Thus, the surface of the sphere is at $r=a$ and the incident plane wave is defined by

$$
\begin{aligned}
E_{x}^{i} & =E_{0} e^{-i k z}=E_{0} e^{-i k r \cos \theta}, \\
H_{y}^{i} & =\frac{E_{0}}{\eta_{0}} e^{-i k z}=\frac{E_{0}}{\eta_{0}} e^{-i k r \cos \theta},
\end{aligned}
$$

for a time factor $\exp (i \omega t)$ and where $\eta_{0}=120 \pi$. The total field can be written as a superposition of TM (transverse magnetic) and TE (transverse electric) waves. Following Harring- 
ton [1961], the field components may be written in terms of potential functions $\boldsymbol{A}$ and $F$, respectively, for the two sets. Explicitly,

$$
\begin{aligned}
E_{r}^{(e)} & =\frac{1}{\sigma+i \epsilon \omega}\left(\frac{\partial^{2}}{\partial r^{2}}+k^{2}\right) A, \\
H_{r}^{(h)} & =\frac{1}{i \mu \omega}\left(\frac{\partial^{2}}{\partial r^{2}}+k^{2}\right) F, \\
E_{\theta}^{(e)} & =\frac{1}{(\sigma+i \epsilon \omega) r} \frac{\partial^{2} A}{\partial r \partial \theta}, \\
H_{\theta}^{(e)} & =\frac{1}{r \sin \theta} \frac{\partial A}{\partial \phi}, \\
E_{\theta}^{(h)} & =-\frac{1}{r \sin \theta} \frac{\partial F}{\partial \phi}, \\
H_{\theta}^{(h)} & =\frac{1}{i \mu \omega r} \frac{\partial^{2} F}{\partial r \partial \theta}, \\
E_{\phi}^{(e)} & =\frac{1}{(\sigma+i \epsilon \omega) r \sin \theta} \frac{\partial^{2} A}{\partial r \partial \phi}, \\
H_{\phi}^{(e)} & =-\frac{1}{r} \frac{\partial A}{\partial \theta}, \\
E_{\phi}^{(h)} & =\frac{1}{r} \frac{\partial F}{\partial \theta}, \\
H_{\phi}^{(h)} & =\frac{1}{(i \mu \omega) r \sin \theta} \frac{\partial^{2} F}{\partial r \partial \phi} .
\end{aligned}
$$

The total field in any region is the superposition of the TM and TE waves. For example,

$$
E_{\theta}=E_{\theta}^{(e)}+E_{\theta}^{(h)} .
$$

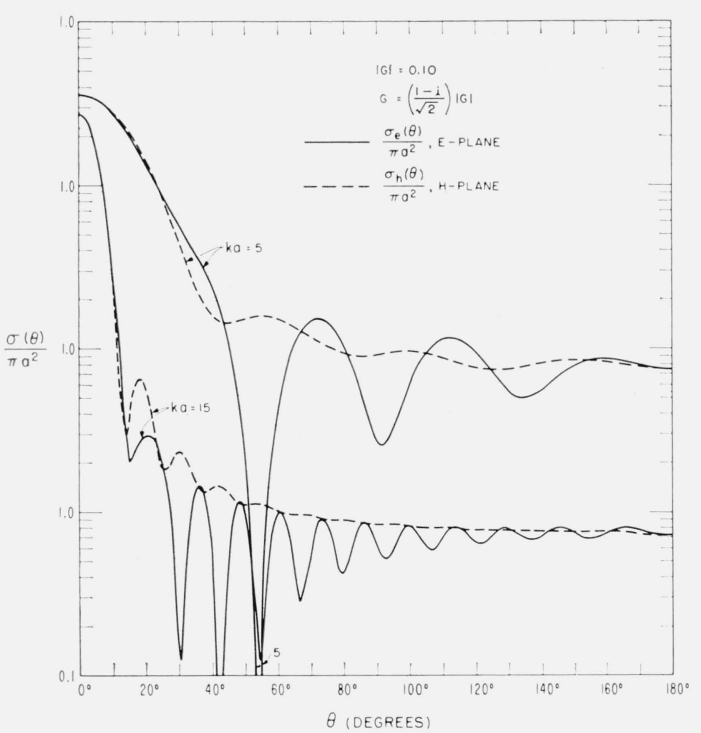

F1GURE 11b.

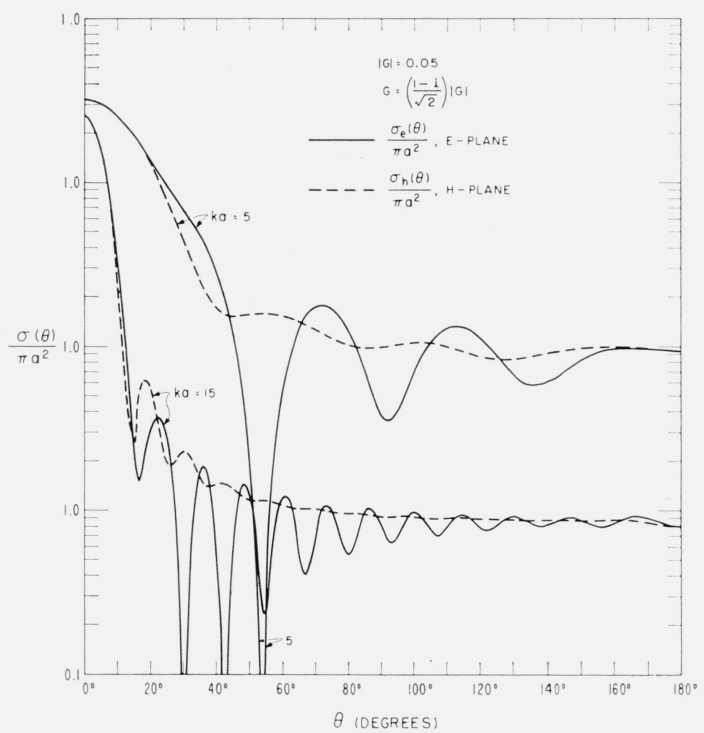

Figure 11c. 


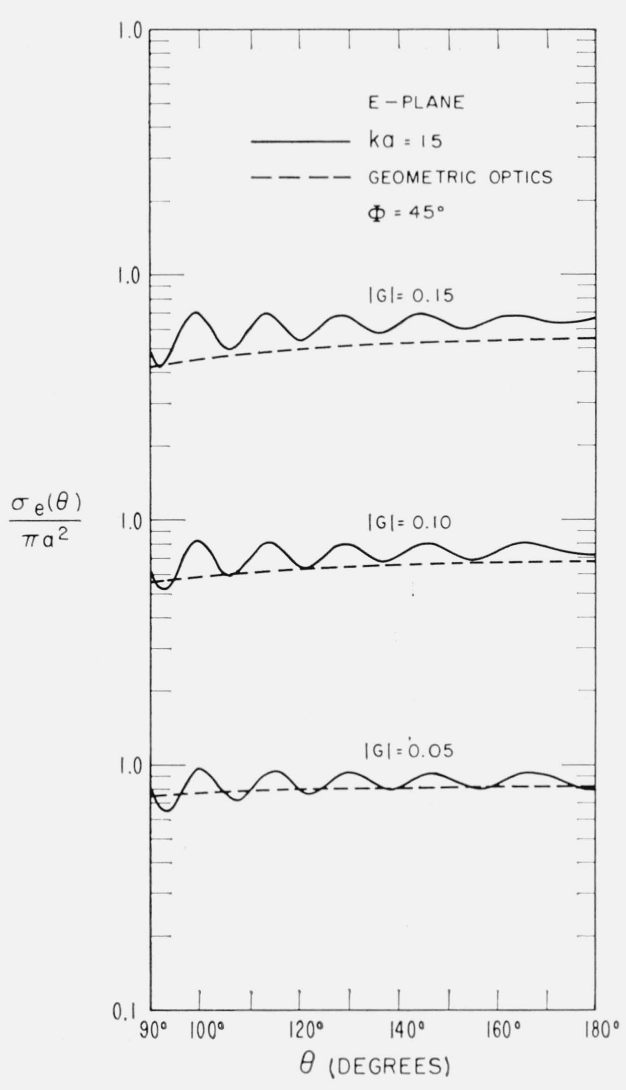

Figure 12a.

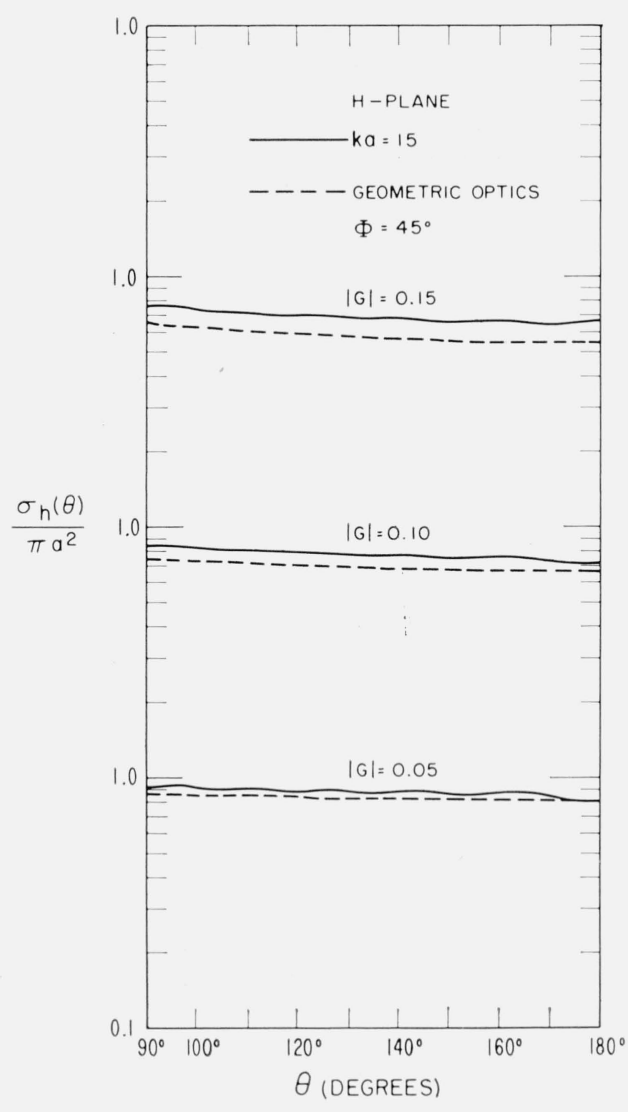

FiguRE $12 \mathrm{~b}$.

The well-known addition theorem [Harrington, 1961],

$$
e^{i k r \cos \theta}=\sum_{n=0}^{\infty} i^{n}(2 n+1) \frac{\hat{J}_{n}(k r)}{k r} P_{n}(\cos \theta),
$$

permits us to write

$$
\begin{aligned}
E_{r}^{i} & =\cos \phi \sin \theta E_{x}^{i}=E_{0} \frac{\cos \phi}{i k r} \frac{\partial}{\partial \theta}\left(e^{-i k r \cos \theta}\right), \\
& =+\frac{i E_{0} \cos \phi}{(k r)^{2}} \sum_{n=1}^{\infty} i^{-n}(2 n+1) \hat{J}_{n}(k r) P_{n}^{1}(\cos \theta),
\end{aligned}
$$

where the notation is that used by Schelkunoff [1943]. $\hat{J}_{n}(k r)$ is a spherical Bessel function defined by

$$
\hat{J}_{n}(k r)=\left(\frac{\pi k r}{2}\right)^{1 / 2} J_{n+1 / 2}(k r),
$$

where $J_{n+1 / 2}$ is a cylindrical Bessel function, $P_{n}^{1}$ is an associated Legendre polynomial defined here by

$$
P_{n}^{1}(\cos \theta)=-\frac{\partial}{\partial \theta} P_{n}(\cos \theta)
$$

in terms of the Legendre polynomial. The potential function $A^{i}$, which gives rise to $E_{r}^{i}$, is easily found to be

$$
A^{i}=-\frac{E_{0} \cos \phi}{\mu \omega} \sum_{n=1}^{\infty} a_{n} \hat{J}_{n}(k r) P_{n}^{1}(\cos \theta)
$$




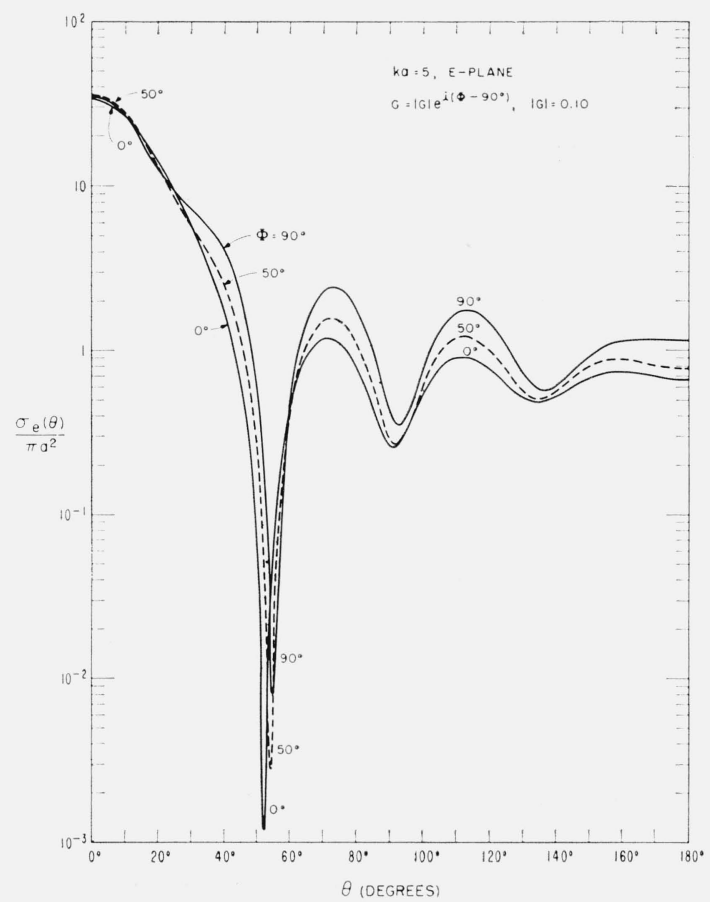

Figure 13a.

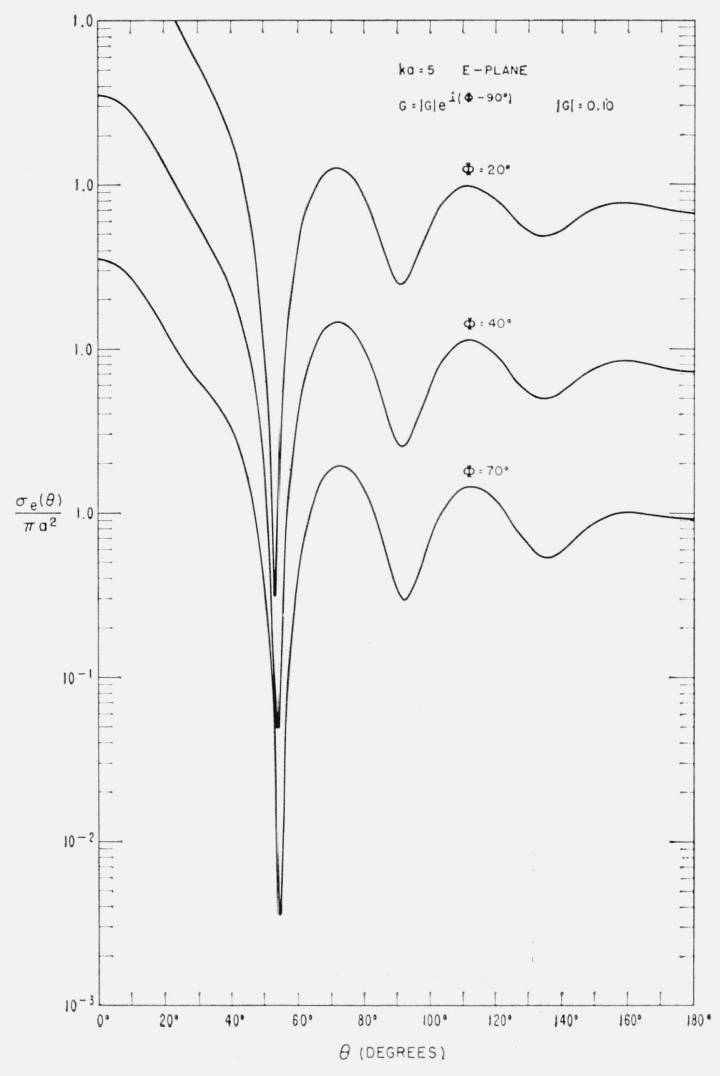

Figure 13 b.

where

$$
a_{n}=\frac{i^{-n}(2 n+1)}{n(n+1)} .
$$

By a similar procedure, it is found that

$$
F^{i}=-\frac{E_{0} \sin \phi}{k} \sum_{n=1}^{\infty} a_{n} \hat{J}_{n}(k r) P_{n}^{1}(\cos \theta) .
$$

For the region $r>a$, it is possible to write

$$
\begin{aligned}
& A=A^{i}+A^{s}, \\
& F=F^{i}+F^{s},
\end{aligned}
$$

where the superscript $s$ is associated with the scattered field.

Clearly, $A^{s}$ and $F^{s}$ must contain the spherical Hankel functions $\hat{H}_{n}^{(2)}(k r)$ in place of the $\hat{J}_{n}(k r)$ 's, because the scattered field must behave as an outgoing wave at infinity. Thus, for the region $r>a$,

and

$$
A=\sum_{n=1}^{\infty} A_{n}=-\frac{E_{0} \cos \phi}{\mu \omega} \sum_{n=1}^{\infty}\left[a_{n} \hat{J}_{n}(k r)+b_{n} \hat{H}_{n}^{(2)}(k r)\right] P_{n}^{(1)}(\cos \theta),
$$

$$
F=\sum_{n=1}^{\infty} F_{n}=-\frac{E_{0} \sin \phi}{k} \sum_{n=1}^{\infty}\left[a_{n} \hat{J}_{n}(k r)+c_{n} \hat{H}_{n}^{(2)}(k r)\right] P_{n}^{(1)}(\cos \theta),
$$

where $b_{n}$ and $c_{n}$ are coefficients unknown as yet. 


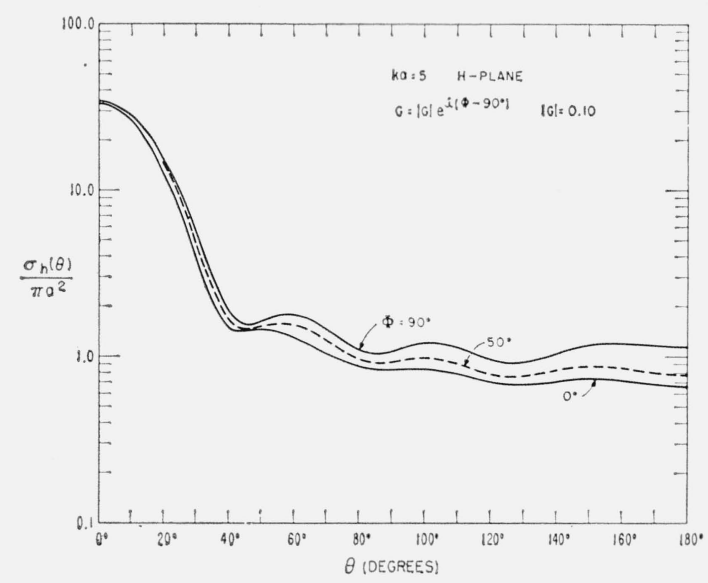

F1GURE 14a.

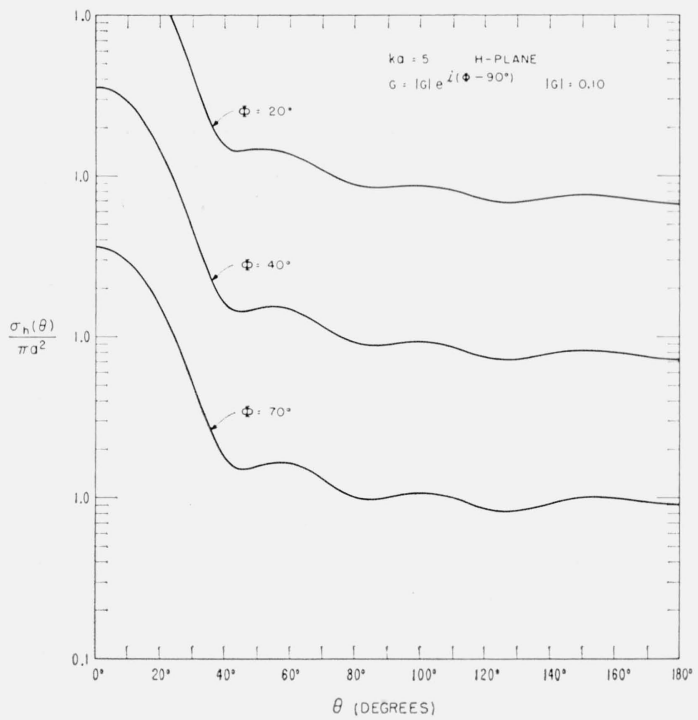

Figure $14 b$.

It is now convenient to introduce the concept of wave impedance such that relations between the tangential field components at $r=a$ are to be prescribed. For example, for a TM mode, of order $n$, we insist that

$$
E_{\theta}^{(e)}=-Z^{(n)} H_{\phi}^{(e)} \text { and } H_{\theta}^{(e)}=\frac{1}{Z^{(n)}} E_{\phi}^{(e)} \text { at } r=a,
$$

while for a TE mode, of order $n$,

$$
H_{\theta}^{(h)}=Y^{(n)} E_{\phi}^{(h)} \text { and } E_{\theta}^{(h)}=-\frac{1}{Y^{(n)}} H_{\phi}^{(h)} \text { at } r=a .
$$

These latter four conditions are automatically satisfied if

and

$$
\left.\frac{1}{i \epsilon_{0} \omega} \frac{\partial A_{n}}{\partial r}=Z^{(n)} A_{n}\right]
$$

Employing the boundary conditions on $A_{n}$ and $F_{n}$, defined above, it is a simple matter to show that

and

$$
b_{n}=+\frac{i^{-n}(2 n+1)}{n(n+1)} B_{n}
$$

where

$$
c_{n}=+\frac{i^{-n}(2 n+1)}{n(n+1)} C_{n},
$$

and

$$
B_{n}=-\frac{\hat{J}_{n}^{\prime}(k a)-i \Delta_{n} \hat{J}_{n}(k a)}{\hat{H}_{n}^{(2) \prime}(k a)-i \Delta_{n} \hat{H}_{n}^{(2)}(k a)},
$$

$$
C_{n}=-\frac{\hat{J}_{n}^{\prime}(k a)-i \delta_{n} \hat{J}_{n}(k a)}{\hat{H}_{n}^{(2) \prime}(k a)-i \delta_{n} \hat{H}_{n}^{(2)}(k a)},
$$

where $k=\left(\epsilon_{0} \mu_{0}\right)^{\frac{1}{2}} \omega, \Delta_{n}=Z^{(n)} / \eta_{0}$, and $\delta_{n}=Y^{(n)} \eta_{0}$. 


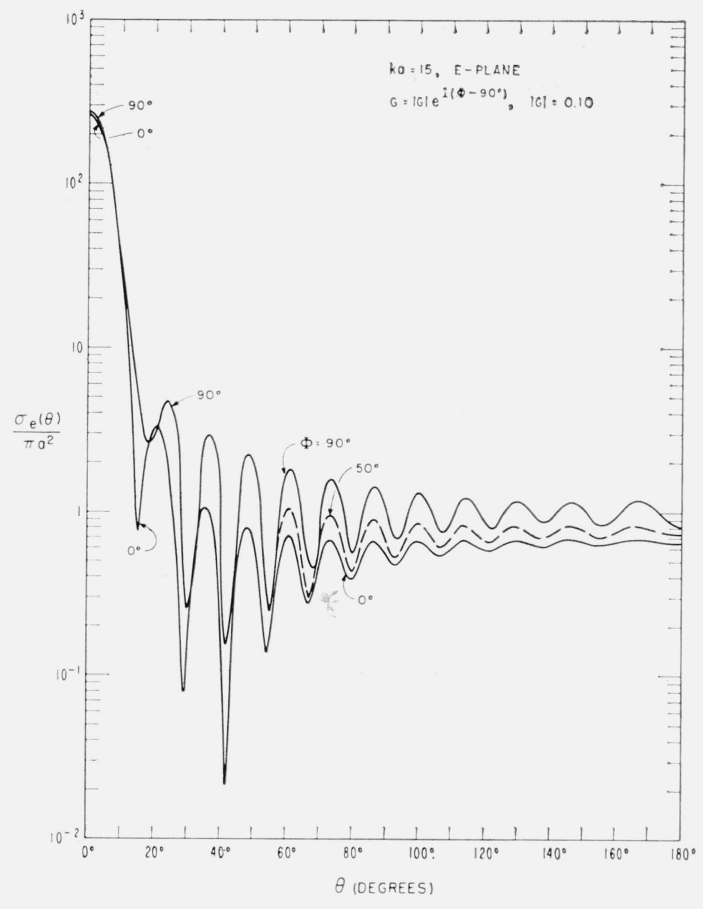

Figure $15 \mathrm{a}$.

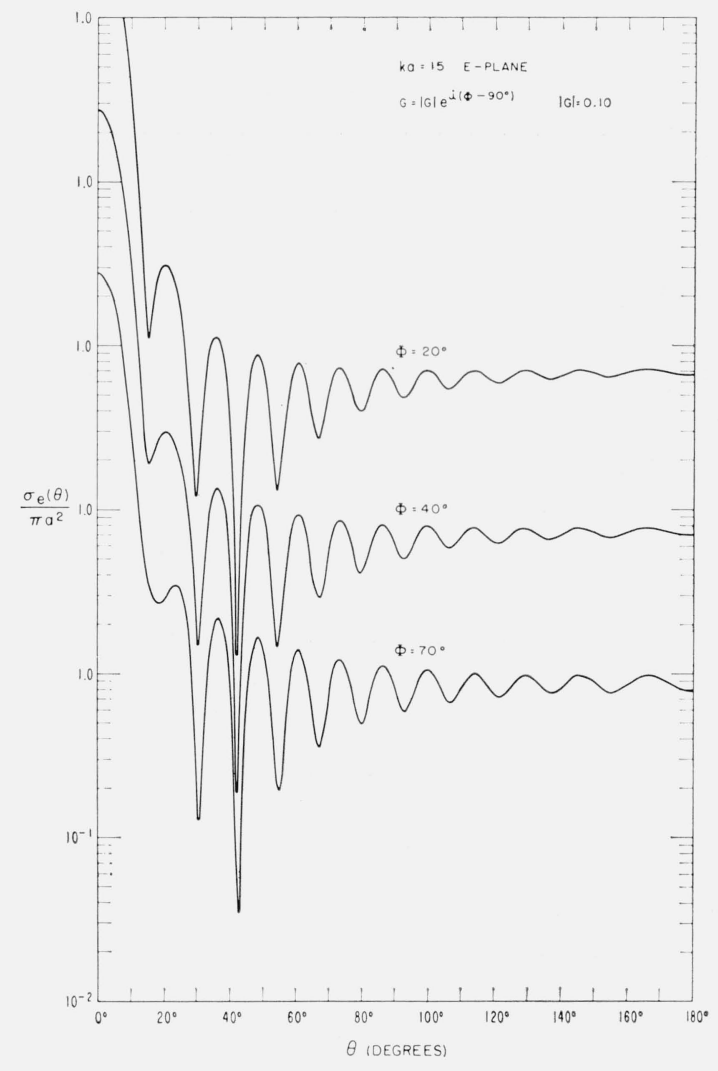

Figure $15 \mathrm{~b}$.

The solution of the first part of the problem has now been obtained. Namely, the fields may be expressed in terms of the normalized impedance $\Delta_{n}$ and normalized admittance $\delta_{n}$. Of particular interest are the far-zone scattered fields such that $k r>>1$. It is then a simple matter to show that

$$
E_{\theta}^{s} \cong+\frac{i E_{0}}{k r} e^{-i k r} \cos \phi P(\theta) \cong \eta_{0} H_{\phi}^{s}
$$

and

where

$$
E_{\phi}^{s} \cong-\frac{i E_{0}}{k r} e^{-i k r} \sin \phi Q(\theta) \cong-\eta_{0} H_{\theta}^{s},
$$

and

$$
P(\theta)=\sum_{n=1}^{\infty} \frac{(2 n+1)}{n(n+1)}\left[B_{n} \frac{d}{d \theta} P_{n}^{1}(\cos \theta)+C_{n} \frac{P_{n}^{1}(\cos \theta)}{\sin \theta}\right]
$$

$$
Q(\theta)=\sum_{n=1}^{\infty} \frac{(2 n+1)}{n(n+1)}\left[B_{n} \frac{P_{n}^{1}(\cos \theta)}{\sin \theta}+C_{n} \frac{d}{d \theta} P_{n}^{1}(\cos \theta)\right]
$$

In order to arrive at an explicit solution, it is necessary to obtain formulas for the impedance $Z^{(n)}$ and the admittance $Y^{(n)}$ for TM and TE modes, respectively. This subject has been discussed in an earlier paper by the author [Wait, 1963] and an equivalent problem has been treated by Garbacz [1962]. For purposes of the present paper, it is assumed that $! Z^{(n)}=1 / Y^{(n)}=Z$ is a specified complex constant independent of mode number $n$. By definition, $Z$ becomes the surface impedance at the boundary $r=a$ as discussed in the main text.

The authors appreciate the extensive advice of K. P. Spies and L. A. Berry in connection with the computing work. In particular, the program for the spherical wave functions, as developed by L. A. Berry, was used extensively. 


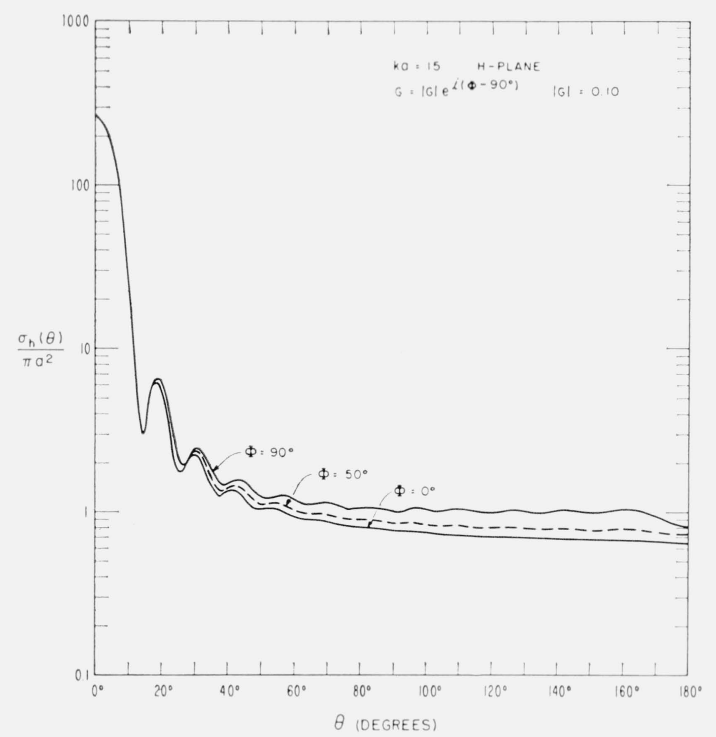

Figure $16 a$.

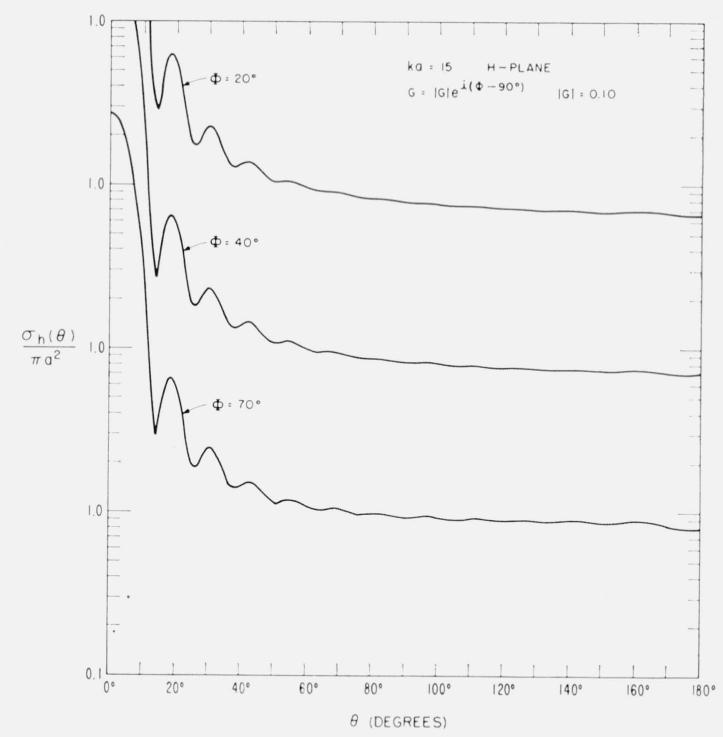

Figure $16 \mathrm{~b}$.

\section{Note Regarding the Figures}

The vertical scale in a number of the figures is shifted in order to avoid overlapping of the curves when the $k a$ value is changed. To prevent confusion, one should remember that $\sigma(\theta)$ tends toward $\pi a^{2}$ when $\theta=180^{\circ}$.

\section{References}

Belkina, M. G., and L. A. Weinstein (1957), Diffraction of electromagnetic waves on certain bodies of rotation (Diffraktsiya elektromagniknykh voln na nekoterylch telakh vrashcheniya), 57-125, Soviet Radio Press, Moscow.

Garbacz, R. J. (Aug. 1962), Electromagnetic scattering from radially inhomogeneous spheres, Proc. IRE 50, 1837 (Letter to the Editor).

Garbacz, R. J. (Jan. 1964), Bistatic scattering from a class of lossy dielectric spheres with surface impedance boundary conditions, Phys. Rev. 133, A14-A16.

Harrington, R. F. (1961), Time harmonic electromagnetic fields (McGraw-Hill Book Co., Inc., New York).

Hiatt, R. E., K. M. Siegel, and H. Weil (Sept. 1960a), The ineffectiveness of absorbing coatings on conducting objects illuminated by long wavelength radar, Proc. IRE 48, 1636-1642.

Hiatt, R. E., K. M. Siegel, and H. Weil (Sept. 1960b), Forward scattering by coated objects illuminated by short wavelength radar, Proc. IRE 48, 1630-1635.

Kerker, M. (editor) (1964), Electromagnetic scattering (Pergamon Press, Oxford).

King, R. W. P., and T. T. Wu (1959), The scattering and diffraction of radio waves (Harvard Monograph in Applied Science, No. 7, Harvard Univ., Cambridge, Mass.).

Logan, N. A., and K. S. Yee (1961), A mathematical model for the theory of diffraction by convex surfaces, Proc. Symposium on Electromagnetic Waves, Publication No. 6, Mathematics Res. Center, University of Wisconsin, Madison).

Miller, J. C. P. (1950), British Association for the Advancement of Science, Bessel Functions, Part I, Functions of order zero and unity (Cambridge Univ. Press, Cambridge).

Schelkunoff, S. A. (1943), Electromagnetic waves (Van Nostrand Co., New York).

Van de Hulst, H. C. (1957), Light scattering by small particles (John Wiley and Sons, Inc., New York).

Wait, J. R. (Apr. 1956), Radiation from a vertical antenna over a curved stratified ground, J. Res. NBS 56, No. 4, 237-244.

Wait, J. R. (1962), Electromagnetic waves in stratified media (Pergamon Press, Oxford).

Wait, J. R. (1963), Electromagnetic scattering from a radially inhomogeneous sphere, Appl. Sci. Res. 10, Sec. B, 441-450.

Wait, J. R. (1964), Electromagnetic surface waves, in Advances in Radio Research (ed. by J. A. Saxton), 1, 157-217 (Academic Press, London).

\section{Additional Reference}

Deirmendjian, D., R. Clasen, and W. Viezee (June, 1961), Mie scattering with complex index of refraction, J. Opt. Soc. Amer. 51, 620-633. 